\title{
Measurement of sub-pulse-width temporal delays via spectral interference induced by weak value amplification
}

\author{
Luis José Salazar-Serrano, ${ }^{1,2}$ Davide Janner, ${ }^{1}$ Nicolas Brunner, ${ }^{3,4}$ Valerio Pruneri, ${ }^{1,5}$ and Juan P. Torres ${ }^{1,6}$ \\ ${ }^{1}$ ICFO-Institut de Ciencies Fotoniques, Mediterranean Technology Park, 08860 Castelldefels (Barcelona), Spain \\ ${ }^{2}$ Physics Department, Universidad de los Andes, AA 4976, Bogota DC, Colombia \\ ${ }^{3}$ Departement de Physique Theorique, Universite de Genève, 1211 Genève, Switzerland \\ ${ }^{4}$ H.H. Wills Physics Laboratory, University of Bristol, BS8 1TL, United Kingdom \\ ${ }^{5}$ ICREA-Institució Catalana de Recerca i Estudis Avançats, 08010 Barcelona, Spain \\ ${ }^{6}$ Universitat Politècnica de Catalunya, Barcelona Tech, \\ Dept. of Signal Theory $\&$ Communications, 08034 Barcelona, Spain
}

\begin{abstract}
We demonstrate experimentally a scheme to measure small temporal delays, much smaller than the pulse width, between optical pulses. Specifically, we observe an interference effect, based on the concepts of quantum weak measurements and weak value amplification, through which a sub-pulsewidth temporal delay between two femtosecond pulses induces a measurable shift of the central frequency of the pulse. The amount of frequency shift, and the accompanying losses of the measurement, can be tailored by post-selecting different states of polarization. Our scheme requires only spectrum measurements and linear optics elements, hence greatly facilitating its implementation. Thus it appears as a promising technique for measuring small and rapidly varying temporal delays.
\end{abstract}

PACS numbers: 03.67.-a, 06.20.-f, 42.50.-p

The measurement of temporal delays between optical pulses is essential in metrology, for instance for accurate distance measurements and for timing synchronization [1, 2], where the capability of discriminating between small temporal delays with a reference pulse is needed. Diverse optical schemes for measuring subpicosecond temporal delays have been demonstrated. This is the case, for instance, of schemes based on the use of ultrafast nonlinear processes such as second harmonic generation [3, 4] or two-photon absorption [5].

In another context, the well-known Hong-Ou-Mandel effect makes use of quantum interference to measure subpicosecond temporal delays between photons [6], which was used by Steinberg et al. [7] for measuring very small single-photon tunneling times. Since this technique is based on measuring two-photon coincidences, it generally restrict the number of photons of the signal. However, quantum-inspired interferometers [8] might broaden the applicability of quantum concepts to other scenarios.

When two similar optical pulses with temporal width $\tau$, and time delay $T \gg \tau$ between them, recombine, a modulation of the spectral density appears 9 [1], which allows measuring the time difference $T$. This is true even if the optical path difference is larger than the coherence length of the pulses [12]. However, for small values of $\mathrm{T}(T \ll \tau)$, inspection of the spectral density reveals no interference effects, even though interference manifest now in the temporal domain as a periodic change of the output intensity as function of the delay.

Here we demonstrate experimentally a scheme to measure small temporal delays $T$ between optical pulses, much smaller than the pulse width $\tau$, based on an interference effect in the frequency domain which produces a measurable shift of the central frequency of the pulse
13. The scheme makes use of linear optics elements only and works in both the high and low signal regimes. It allows the measurement of temporal delays between optical pulses up to the attosecond timescale [14]. This phenomenon, which is inspired by the concepts of quantum weak measurements and weak value amplification 15 18], produces interference effects in the regime $T \ll \tau$, which allows to deduce the value of $T$.

Although the concept of weak measurements originates from research on quantum theory, the phenomenon of weak value amplification can be readily understood in terms of constructive and destructive interference between waves [16, 19, 20]. In a weak measurement scenario, a system is weakly coupled to a pointer (the measuring device). While the weakness of the coupling can be seen as a disadvantage at first sight, Aharonov and colleagues [15] showed that when appropriate initial and final states of the system are selected (i.e. pre- and postselection), the pointer is shifted by an unexpectedly large amount. It was soon suggested that these ideas may find application in metrology [17, 18]. This phenomenon, termed weak value amplification, have been demonstrated experimentally [21 23], and have been used for measurements of very small transverse displacements of optical beams [24, 25], as well as for frequency [26] and velocity measurements 27]. Techniques for measuring small phase shifts have also been proposed [13, 14, 28].

Here, the weak coupling is realized by means of a polarization-dependent temporal delay implemented in a Michelson interferometer configuration (see Fig. 1). Brunner and Simon [13] showed that the introduction of a small temporal delay between the two components (horizontal and vertical) of a circularly-polarized pulse, can yield a large central frequency shift after recombin- 


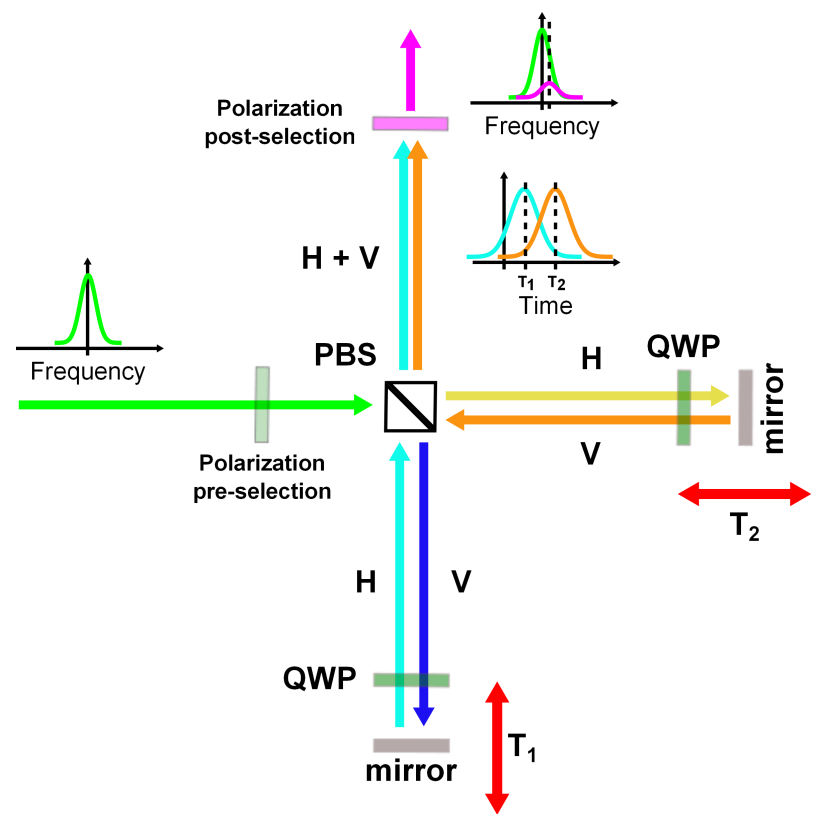

FIG. 1. Schematic of the experimental setup. State preselection: The polarization of the input optical pulse is selected by using $\lambda / 2$ and $\lambda / 4$ wave-plates (not shown). Weak coupling: A Michelson-Morley interferometer, composed of a Polarizing Beam Splitter (PBS), two $\lambda / 4$ wave-plates and two mirrors, divides the input pulse into two pulses, with equal power and with orthogonal polarizations, that travel through different paths of the interferometer. A movable mirror mounted on a translation stage in one of the paths allows changing the temporal delay. State post-selection: The two pulses recombine in the PBS, and they are projected into a particular state of polarization with a electrically-controlled liquid crystal variable retarder (LCVR) and a polarizer. The output beam is finally focused in a single mode fiber (SM) and its spectrum is measured with an Optical Spectrum Analyzer (OSA).

ing the pulses and projecting them into a polarization state nearly orthogonal to the input state. However, the near orthogonality of the input and output polarization states introduces heavy losses. Nevertheless, the weak value amplification can also be used when the input and output polarization states have a relatively large overlap, hence away from the usual weak value amplification regime [20], allowing for the observation of significant frequency shifts without heavy losses, as we will demonstrate here.

Recently, it has been demonstrated that high precision phase estimation based on weak measurements can be achieved even using commercial light-emitting diodes [29]. Indeed, $\mathrm{Li}$ et al. [28] showed that the scheme proposed by Brunner and Simon also works with largebandwidth incoherent light. On the one hand, the use of white light allows to obtain in a straightforward manner a light source with an enormous bandwidth, which allows to measure very small phase differences. On the other hand, many applications make use of high-repetition femtosecond sources that allows to perform multiple measurements in millisecond or microsecond time intervals [2], allowing the measurement of time-varying phase differences in this time scale. This is the scenario that we consider here.

We make use of a femtosecond fiber laser (Calmar Laser - Mendocino) centered at $1549 \mathrm{~nm}$ (temporal width: $320 \mathrm{fs}$; average power: $3 \mathrm{~mW}$; repetition rate: $20 \mathrm{MHz}$ ). The spectral density measured shows characteristic high-frequency small wrinkles due to cavity effects in the laser system. The spectral density is $S_{i n}(\nu)=$ $1 / 2 \epsilon_{0} c\left|E_{\text {in }}(\nu)\right|^{2}$, where $E_{\text {in }}(\nu)$ is the electric field, $\nu$ designates the frequency, $\epsilon_{0}$ is the vacuum permittivity and $c$ is the velocity of light. The input optical pulse is preselected to be left-handed circularly polarized, with polarization vector $\mathbf{e}_{i n}=(\mathbf{x}-i \mathbf{y}) / \sqrt{2}$. A polarizing beam splitter (PBS) divides the input pulse into two orthogonally linearly polarized components with horizontal $(\mathbf{x})$ and vertical (y) polarizations, which propagate along the two arms of a Michelson interferometer. By changing the length of each arm, $d_{1}$ and $d_{2}$, we introduce different time delays $T_{1}=2 d_{1} / c$ and $T_{2}=2 d_{2} / c$ for each polarization component. The two delayed pulses recombine at the same PBS. Finally, in the post-selection stage, the outgoing pulse is projected into a state of polarization given by the polarization vector $\mathbf{e}_{\text {out }}=[\mathbf{x}+\exp (i \Gamma) \mathbf{y}] / \sqrt{2}$, where $\Gamma$ determines the final state of polarization of the output pulse. For $\Gamma=-\pi / 2$, the input and output polarization states coincide, while for $\Gamma=\pi / 2$, they are orthogonal. The polarization of the output beam is post-selected with a Liquid Crystal Variable Retarder (LCVR) (Thorlabs - LCC1113-C) followed by a polarizer. The relation between post-selection angle and the LCVR voltage is non-linear and highly temperature dependent. For this reason, an additional temperature controller is used. After the polarization post-selection, the electric field of the output signal writes

$$
E_{\text {out }}(\omega)=\frac{E_{\text {in }}(\omega)}{2}\left[\exp \left(i \omega T_{1}\right)-i \exp \left(i \omega T_{2}-i \Gamma\right)\right],
$$

where $\omega=2 \pi \nu$. Eq. (1) shows that the post-selection polarization state $(\Gamma)$ determines for which frequencies the interference between signals coming from the horizontally and vertically polarized pulses, delayed by $T=T_{1}-T_{2}$, is constructive or destructive.

We measure the output spectral density which is given by

$$
S_{\text {out }}(\nu)=\frac{S_{\text {in }}(\nu)}{2}[1+\cos (2 \pi \nu T-\Gamma-\pi / 2)] .
$$

where $S_{i n}(\nu)$ is the laser spectrum. In order to characterize the output spectrum, we measure as a function of the post-selection angle $\Gamma$, the central frequency shift $\Delta f=\int d \nu \nu\left[S_{\text {out }}(\nu)-S_{\text {in }}(\nu)\right]$ and the insertion 
(a)

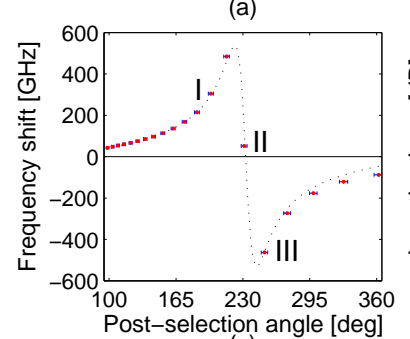

(c)

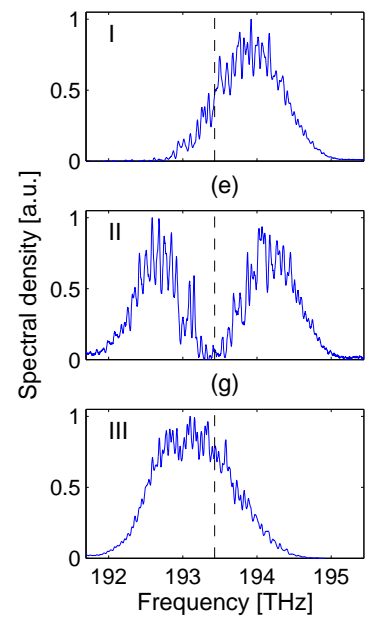

(b)

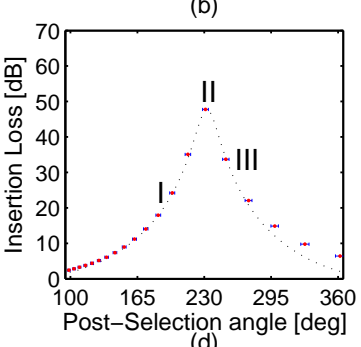

(d)
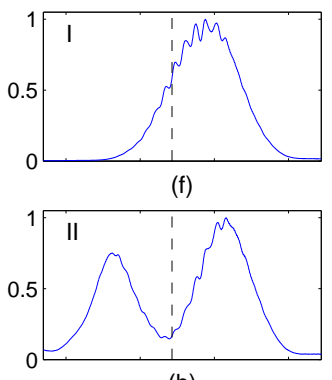

(h)

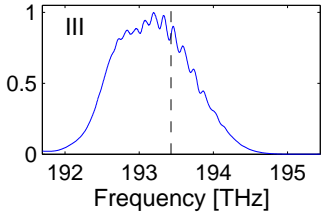

(i)

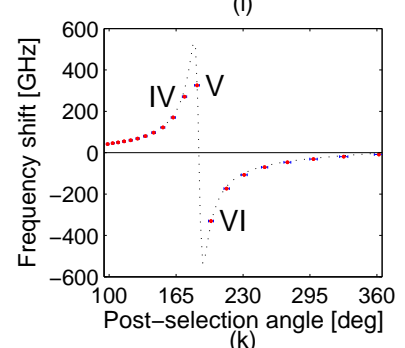

(k)
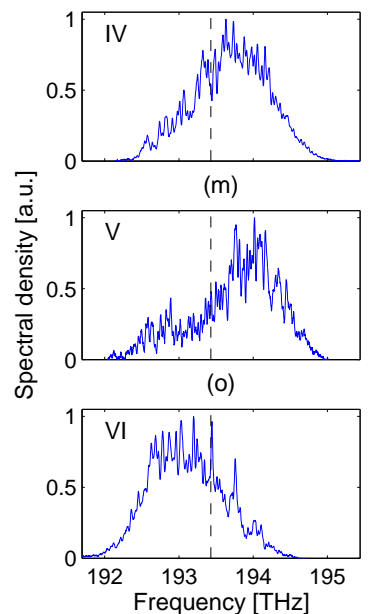

(j)
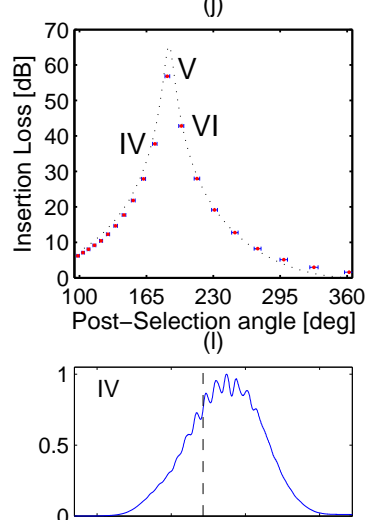

(n)

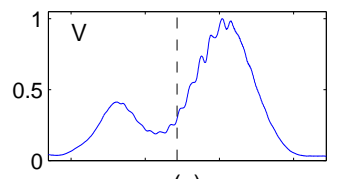

(p)

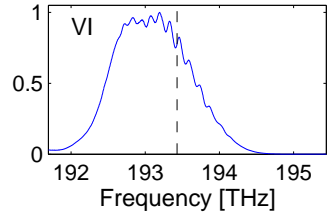

FIG. 2. Measurement of the central frequency shift induced by weak value amplification. Measured frequency shift (a) and insertion loss (b) as a function of the post-selection angle $\Gamma$. Dots (with error bars) are experimental results, and the dotted lines are best theoretical fits using the measured input spectrum in Eq. 2. The best fits are obtained for $T=53 \mathrm{fs}$ in (a) and (b), and $T=22 \mathrm{fs}$ in (i) and (j). For $T=53 \mathrm{fs}$ : (c), (e) and (g) (measured) and (d), (f) and (h) (theory) shows the spectral density for some selected cases, as indicated by the corresponding labels in (a) and (b). For $T=22 \mathrm{fs}$ : (k), (m) and (o) (measured) and (l), (n) and (p) (theory) shows the spectral density for some selected cases, as indicated by the corresponding labels in (i) and $(\mathrm{j})$. To help the eye, the central frequency of the input pulse $\left(\nu_{0}=193.44 \mathrm{THz}\right)$ is represented by a dashed line in all plots. The experiment is performed at a temperature of $34.1^{\circ} \mathrm{C}$. Error bars in all plots assume that temperature variations during the experiment are in the range of $\pm 1^{\circ} C$, which translates in random changes of the angle of post-selection $\Gamma$.

$\operatorname{loss} L=-10 \log F_{\text {out }} / F_{\text {in }}$, with $F_{\text {in,out }}$ being the input (output) energy $F_{\text {in,out }}=\int_{-\infty}^{\infty} S_{\text {in,out }}(\nu) d \nu$ of the pulse. The Optical Spectrum Analyzer (Yokogawa AQ6370) has a resolution of $0.02 \mathrm{~nm}$. Each spectrum is obtained after averaging five data sets in the interval [191.5 THz, 195.5 THz].

Fig. 2 shows measurements of the spectral changes in the regime $T \ll \tau$, when one makes use of the idea of weak value amplification. It shows the shift of the central frequency of the spectrum for two different temporal delays: $T=53$ fs and $T=22$ fs. Fig. 2(a) shows the measured frequency shift and Fig. 2(b) plots the measured insertion loss for $T=53 \mathrm{fs}$ (similarly Figs. 2(i) and 2(j) for $T=22 \mathrm{fs}$ ). The dotted lines are best theoretical fits using the measured input spectrum in Eq. 2 , All other plots in Fig. 2 show measured spectral densities of the output signal for some selected cases, and the corresponding theoretical predictions when the measured input spectral density is used in Eq. (2).

Inspection of Fig. 2 allows to highlight two working regimes, corresponding to the presence of high or low losses. For $\Gamma=-3 \pi / 2+2 \pi \nu_{0} T$, there is no central frequency shift and losses are maximum. The output spectral density features a double-peak spectral density. For small angle deviations around this value, central frequency shifts of the spectral density up to hundreds of gigahertz are clearly observable. However, insertion losses are also the highest in this regime, measuring values over $60 \mathrm{~dB}$. This regime corresponds to the case usually studied in weak value amplification where the input and output polarization states are nearly orthogonal [13]. The applicability of the weak value amplification in this highamplification regime is limited to cases where the energy of the input signal can be increased, since the intensity of the detected signal is severely decreased 24].

Nevertheless, we demonstrate here that even in the regime where the input and output polarization states have a significant overlap - hence featuring smaller insertion losses - weak value amplification remains useful. Even though the frequency shifts measured in this regime are generally smaller - reaching only few tens of $\mathrm{GHz}$ instead of hundreds of $\mathrm{GHz}$ - losses do not exceed a few 

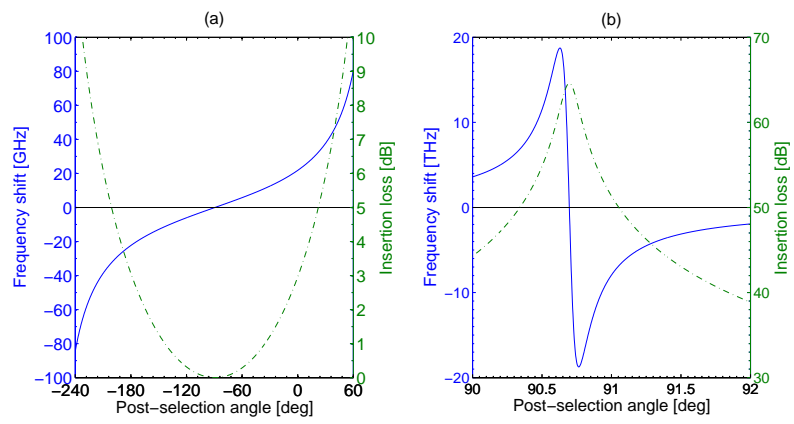

FIG. 3. Feasibility of the measurement of attosecond temporal delays with femtosecond pulses. Polarization dependent frequency shift induced by a $T=10$ time delay of pulses of duration $\tau=10$ fs. (a) Low-loss and (b) High-loss regime. The solid line (blue) corresponds to the frequency shift and the dashed (green) line to the insertion losses. Notice the difference scales in the $x$ and $y$ axis in (a) and (b).

$\mathrm{dB}$. For $\Gamma=-\pi / 2+2 \pi \nu_{0} T$, there is no shift of the central frequency again. The pre- and post-selected polarizations are almost equal, hence introducing almost no losses. The spectral density of the output pulse is almost equal to the input spectral density. For small angle deviations around this value, the temporal delay produce small shifts of the central frequency, which vary almost linearly with respect to the post-selection angle. Importantly, these frequency shifts are accompanied by small insertion losses.

In general, there is a trade-off between the frequency shift observable for a specific value of the time delay and the amount of losses that can be tolerated to keep a good signal-to-noise ratio. The existence of the low-loss working regime, somehow not so extensively considered as the high-loss regime, can thus enhance the applicability of the weak value amplification idea, as demonstrated here.

The results presented here naturally raise the question of what are the ultimate limits of the scheme, in terms of central frequency shifts and losses. Brunner et al. 13. and Strubi et al. 14 have estimated theoretically that weak value amplification of temporally delayed optical pulses could allow the measurement of attosecond temporal delays. Indeed, Xu. et al. 29] have demonstrated the measurement of phase differences as small as $\Delta \varphi \sim 10^{-3}$, which corresponds to an optical path delay difference of $d=\lambda /(2 \pi) \Delta \varphi \sim 130 \mathrm{pm}$, by using a large bandwidth LED source. In principle, one can always make use of white light sources with bandwidths in excess of $100 \mathrm{~nm}$, as the ones use in Optical Coherence Tomography for submicron resolution [30], to enhance the frequency shift detected.

Let us consider as example an input optical pulse with a Gaussian spectrum, i.e., $S_{i n}(\nu) \propto \exp \left[-\pi^{2} \tau^{2}(\nu-\right.$ $\left.\left.\nu_{0}\right)^{2} / \ln 2\right]$, where $\tau$ is the pulse temporal width (FWHM). The central frequency shift $\Delta f$ of the output pulse can be easily calculated and yields

$$
\Delta f=-\frac{\ln 2}{\pi}\left(\frac{T}{\tau^{2}}\right) \frac{\gamma \sin \left(2 \pi \nu_{0} T-\Gamma-\pi / 2\right)}{1+\gamma \cos \left(2 \pi \nu_{0} T-\Gamma-\pi / 2\right)},
$$

where $\gamma=\exp \left[-\ln 2 T^{2} / \tau^{2}\right]$. The frequency shift given by Eq. (3) is accompanied by insertion losses which write

$$
L=-10 \log \left[\frac{1}{2}\left(1+\gamma \cos \left(2 \pi \nu_{0} T-\Gamma-\pi / 2\right)\right)\right] .
$$

Fig. 3 shows the frequency shift expected, as a function of the post-selection state of polarization, when a 10 as temporal delay is introduced between two optical pulse with duration $\tau=10 \mathrm{fs}$.

Fig. 3(a) depicts the low-loss regime, where smaller frequency shifts can be observed in exchange for much lower losses. In the case shown, frequency shifts up to $100 \mathrm{GHz}$, corresponding to $0.8 \mathrm{~nm}$, are generated with losses below $12 \mathrm{~dB}$. Most spectrometers, as the one used in our experiments, can reach resolutions of up to 0.02 $\mathrm{nm}$, rendering measurable these frequency shifts. In the high-loss regime, shown in Fig. 3(a), one can observe greater frequency shifts, as high as $\sim 20 \mathrm{THz}(\sim 160 \mathrm{~nm})$. Unfortunately, its measurement is also accompanied by higher losses, over $60 \mathrm{~dB}$.

In conclusion, we have demonstrated a spectral interference effect between two optical pulses with a temporal delay much smaller than the pulse duration, inspired from the concepts of weak measurements and weak value amplification. In particular, we have demonstrated a shift of the central frequency of two slightly delayed femtosecond pulses which can be used to reveal the value of the temporal delay itself. Importantly, the central frequency shifts can be observed even in a regime, not sooften considered, where insertion losses are small, which broadens the applicability of the method demonstrated.

Our scheme is implemented by using only linear optics elements and requires spectral measurements, hence making its implementation practical.The ultimate sensitivity of our scheme can provide observable frequency shifts for temporal delays of the order of attoseconds using femtosecond laser sources. Our scheme thus appears as a promising method for measuring small and rapidly varying temporal delays.

Acknowledgements: We acknowledge support from the EU project PHORBITECH (FET OPEN grant number 255914), the Spanish government projects FIS2010-14831, TEC201014832, the Ramon y Cajal and Severo Ochoa programs, and Fundació Privada Cellex, Barcelona. LJSS acknowledges support from the "Fundación Mazda para el Arte y la Ciencia", Bogotá, Colombia, and NB from the Swiss National Science Foundation (grant PP00P2_138917).

[1] J. Kim, J. A. Cox, J. Chen and F. X. Kartner, Nat. Photon. 2, 733 (2008). 
[2] J. Lee, Y-J. Kim, K. Lee, S. Lee and S-W. Kim, Nat. Photon. 4, 716 (2010).

[3] R. Trebino, Nat. Photon. 5, 185 (2005).

[4] A. Peer, B. Dayan, A. A. Friesem and Y. Silberberg, Phys. Rev. Lett. 94, 073601 (2005).

[5] F. Boitier, A. Godard, E. Rosencher and C. Fabre, Nat. Phys. 5, 267 (2009).

[6] C. K. Hong, Z. Y. Ou and L. Mandel, Phys. Rev. Lett. 59, 2044-2036 (1987).

[7] A. M. Steinberg, P. G. Kwiat and R. Y. Chiao, Phys. Rev. Lett. 71, 708 (1993).

[8] R. Kaltenbaek, J. Lavoie, D. N. Biggerstaff and K. J. Resch, Nat. Phys. 4, 864 (2008).

[9] W. P. Alford and A. Gold, Am. L. Phys. 26, 481 (1958).

[10] M. P. Givens, J. Opt. Soc. Am. Phys. Rev. Lett. 51, 1030 (1961).

[11] L. Mandel, J. Opt. Soc. Am. 52, 1335 (1962).

[12] X. Y. Zou, T. P. Grayson ansd L. Mandel, Phys. Rev. Lett. 69, 3041 (1992).

[13] N. Brunner and C. Simon, Phys. Rev. Lett. 105010405 (2010).

[14] G. Strubi and C. Bruder, Phys. Rev. Lett. 110083605 (2012).

[15] Y. Aharonov, D. Z. Albert, and L. Vaidman, Phys. Rev. Lett. 60, 1351 (1988).

[16] I. M. Duck, P. M. Stevenson, and E. C. G. Sudarhshan, Phys. Rev. D 40, 2112 (1989).

[17] Y. Aharonov and L. Vaidman, Phys. Rev. A 41, 11-20 (1990).
[18] J. Dressel, M. Malik, F. M. Miatto, A. N. Jordan, R. W. Boyd, arXiv:1305.7154 [quant-ph].

[19] J. C. Howell, D. J. Starling, P. B. Dixon, K. P. Vudyasetu, and A. N. Jordan, Phys. Rev. A 81, 033813 (2010).

[20] J. P. Torres, G. Puentes, N. Hermosa, and L. J. SalazarSerrano, Opt., Express 2018869 (2012).

[21] N. W. M. Ritchie, J. G. Story, J. G. and R. G. Hulet, Phys. Rev. Lett. 66, 1107 (1991).

[22] N. Brunner, V. Scarani, M. Wegmüller, M. Legré, N. Gisin, Phys. Rev. Lett. 93, 203902 (2004),

[23] G. J. Pryde, J. L. O'Brien, A. G. White, T. C. Ralph, and H. M. Wiseman, Phys. Rev. Lett. 94, 220405 (2005).

[24] O. Hosten and P. Kwiat, Science 319, 787 (2008).

[25] P. Ben Dixon, D. J. Starling, A. N. Jordan, and J. C. Howell, Phys. Rev. Lett. 102, 173601 (2009).

[26] David J. Starling, P. Ben Dixon, A. N. Jordan, and J. C. Howell, Phys. Rev A 82, 063822 (2010).

[27] G.I. Viza, J. Martinez-Rincon, G. A. Howland, H. Frostig, I. Shomroni, B. Dayan, and J. C. Howell, preprint arXiv:1304.0029 (2013).

[28] C.-F. Li, X.-Y. Xu, J.-S. Tang, J.-S. Xu, G.-C. Guo, Phys. Rev. A 83, 044102 (2011).

[29] X.-Y. Xu, Y. Kedem, K. Sun, L. Vaidman, C.-F. Li and G.-C. Guo, Phys. Rev. Lett. 111, 033604 (2013).

[30] B. Povazay, K. Bizheve, A. Unterhuber, B. Hermann, H. Sattmann, A. F. Fercher, W. Drexler, A. Apolonski, W. J. Wadsworth, J. C. Knight, P. St. J. Russell, M. Vetterlein, and E. Scherzer, Opt. Lett. 27, 1800 (2002). 